\title{
LIAM- Logistic and Intelligent Agricultural model for Phytopathology Identification
}

\author{
Joel S Joy ${ }^{1}$, Rroan $M^{2}$, Jithin A M ${ }^{3}$, Ms. Neethu Krishna ${ }^{4}$ \\ ${ }^{1}$ Student, Dept. of Computer Science \& Engineering, Mangalam College of \\ Engineering India, joelsjoyt@ gmail.com \\ ${ }^{2}$ Student, Dept. of Computer Science \& Engineering, Mangalam College of \\ Engineering,India, rroanmoorthy1998@gmail.com \\ ${ }^{3}$ Student, Dept. of Computer Science \& Engineering, Mangalam College of Engineering, \\ India, jithinam741@ gmail.com \\ 4 Assistant Professor, Dept. of Computer Science \& Engineering, Mangalam College of \\ Engineering, India, neethukrishnakadaplackal@gmail.com
}

\begin{abstract}
In agricultural sector the most challenging that farmers faced are the pests that affect the daily crops. Thus, farmers can find the diseased plant for up to a limit. Sometimes they don't even recognize with their bare eyes. So, in order to avoid all these things,a model is created named LIAM (Logistic \&Intelligent Agricultural Model) to classify these diseased plants and predict the disease that are commonly occurred to plants. This model is based on Machine learning (ML) and will classify the desired plant into several layers as it uses Convolutional Neural Network (CNN) which basically, takes a part of a matrix and iterate into several layers. Thereby as we train this model as much as we can it will work as better efficiency and also normally this will basically be open source but as for farmers or home users may not know these technologies to use. So, we have an additional interface to link the model with that, so as to input the data into it to get the desired result.Finally, the model will predict the disease as we input the data with top accuracy.
\end{abstract}

Key words: Artificial Intelligence (AI), Convolutional Neural Network (CNN), Machine Learning (ML), Pytorch.

\section{INTRODUCTION}

\subsection{Machine Learning Overview}

Machine Learning is nothing but a machine learning by itself and performs way better than a human performance. Here we need to train a model as much as we can in order to gain the maximum accuracy and thereby machine will itself learn when a data is given into. Many software tools can be used to do Machine Learning such as Tensorflow,Keras,Pytorch etc.

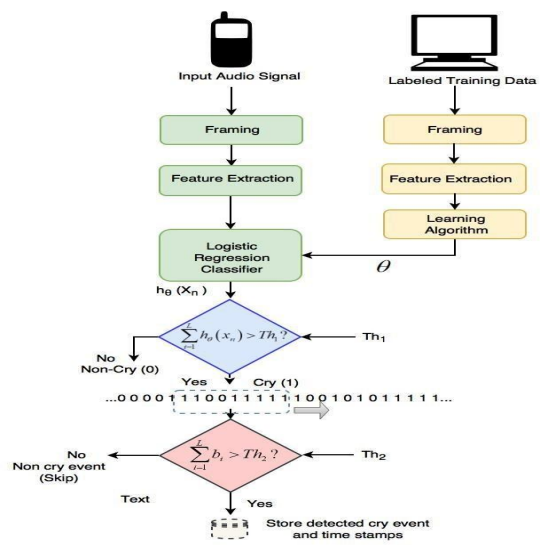

Figure 1: ML Block Diagram

So as per the figure1, it absolutely reflects that our model needs an input of around 1,50,000 images of plant and these inputs will be given and based on that data's are split into train and validation set.

Then it will extract the required features and converted into vector forms in order for the machine learning to perform [10]. Then it will take a part of the image and started to do Convolutional Neural Network in which that particular portion will be off vector forms and those vector form will be taken and iterated into several layers to gain the output.

The specialty of CNN is that it is one of the parts of Neural Networks where it contains $\mathrm{N}$ layers and each layer output will be an efficient one. Here as we are extracting features in all layers, some of them may be lose or not even considered. But this 
CNN have a specialty of back propagation to unwanted features that we left [9].During back propagation the unwanted features are again iterated into layers by layers to gain the output so that no part of features is left and finally we can classify the input and produce the output contain what disease that the desired plant has.

\subsection{CNN Overview}

Convolutional Neural Networks (CNN) is one of the advanced and efficient technique used in classification process. This method takes an input image in the format of vector and takes a unit cell of that image and started to iterate different layers of that image, called convolutional layers [1]. This layer again undergoes several layers and further produce the output. This will go in several neural patterns. Finally getting a classified version (Figure 2).

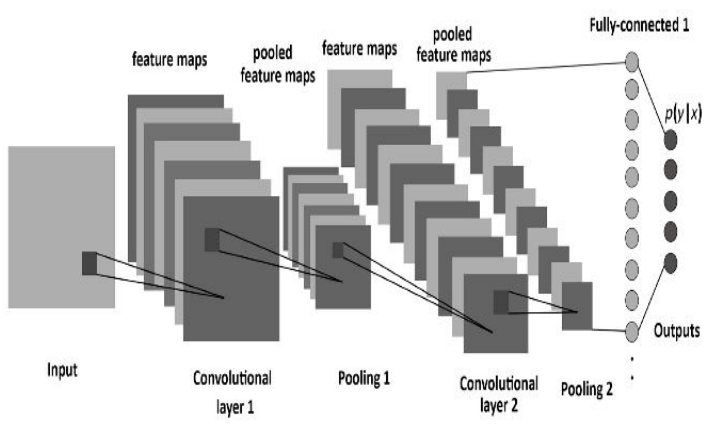

Figure 2: CNN Block Diagram

\section{MOTIVATION}

As of advancement in technologies is getting wider our CNN will clearly classifies finely and gives us a delicate and accurate output. Since we have lots of old-fashioned technologies for classification but by using latest technologies and when this get applied to important and needy field like agriculture simplifies the way of manual power of farmers and also can be applied to all other people [1].

Since farmers doesn't know this much of technologies and also for the other users will much difficult if we just have an open source type. Instead our model will also be linked to an interface in such a way that, for a farmer he can give to some respective area for check if his desired crop have a disease or not and for other users like those who undertake their own vegetable garden can use this interface as such they just want give the input to obtain the desired result.

\section{LITERATURE SURVEY}

Table 1: Literature review

\begin{tabular}{|c|c|c|c|}
\hline SL NO. & TITLE & PROS & CONS \\
\hline 1 & $\begin{array}{l}\text { An image processing } \\
\& \text { neural network- } \\
\text { based approach for } \\
\text { detection \& } \\
\text { classification of } \\
\text { plant leaf diseases }\end{array}$ & $\begin{array}{l}\text { blight, late blight, } \\
\text { powdery-mildew and } \\
\text { Septoria are the } 4 \\
\text { features which they } \\
\text { classified }\end{array}$ & $\begin{array}{l}\text { - Procedures are large } \\
\text { - Time consuming } \\
\text { when taken for } \\
\text { iterations }\end{array}$ \\
\hline 2 & $\begin{array}{l}\text { Detection of } \\
\text { unhealthy region of } \\
\text { plant leaves and } \\
\text { classification of plant } \\
\text { leaf diseases using } \\
\text { texture features }\end{array}$ & $\begin{array}{l}\text { Classified based on } \\
\text { texture features }\end{array}$ & $\begin{array}{l}\text { It doesn't get that } \\
\text { much accuracy when } \\
\text { classified with texture } \\
\text { feature }\end{array}$ \\
\hline 3 & $\begin{array}{l}\text { Fast and Accurate } \\
\text { Detection and } \\
\text { Classification of Plant } \\
\text { Diseases }\end{array}$ & & $\begin{array}{l}\text {-Give only accuracy } \\
\text { of } 84 \% \\
\text {-Uses heavy } \\
\text { mathematical } \\
\text { equation }\end{array}$ \\
\hline 4 & $\begin{array}{l}\text { Image Thresholding } \\
\text { Using Weighted } \\
\text { Parzen-Window } \\
\text { Estimation }\end{array}$ & & $\begin{array}{l}\text {-Clustering based on } \\
\text { pixels and weights } \\
\text {-Uses heavy } \\
\text { mathematical } \\
\text { equation }\end{array}$ \\
\hline 5 & $\begin{array}{l}\text { Implementation of } \\
\text { RGB and Grayscale } \\
\text { Images in Plant } \\
\text { Leaves Disease } \\
\text { Detection }\end{array}$ & & $\begin{array}{l}\text {-It gives o/p of RGB } \\
\text { is better for } \\
\text { classification of } \\
\text { identifying disease } \\
\text { - Accuracy is low }\end{array}$ \\
\hline
\end{tabular}

Table1 is comparison of LIAM with other techniques used for prediction of plant diseases happening in agricultural fields. The review is about the technique and accuracy of that model to predict the desired disease.

Other techniques use different procedure either in different step by step or by mathematical steps in order to attain the result and also accuracy wise each have a variation of accuracy but in our model, we used latest technology to classify the disease [5].

\section{PROPOSED APPROACH}

The proposed model named LIAM- Logistic and Intelligent Agricultural Model used for identification of phytopathology. Here it's a Machine Learning model trained to reach top accuracy. This model contains a dataset of 1,50,000 images from the Kaggle dataset. This dataset undergoes several processes called splitting of data, the architectural model or Deep learning model, Train and validate model, visualisation, finally producing he desired output. 
Joel S Joy et al ., International Journal of Advances in Computer Science and Technology, 9(6), June 2020, 5 - 9

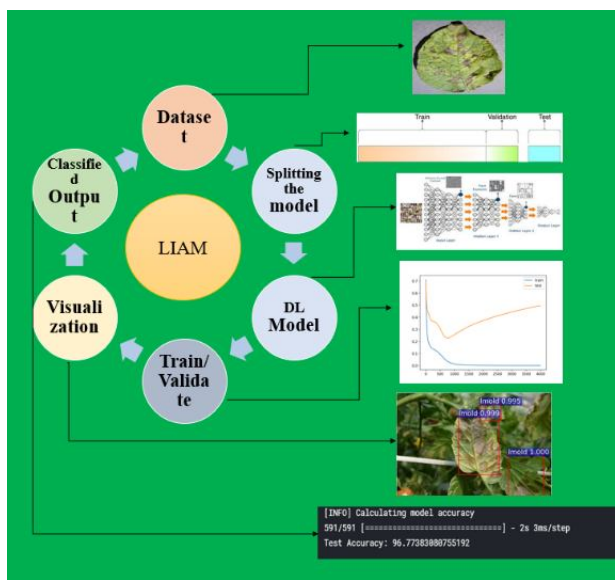

Figure 3: Block Diagram of LIAM

In Figure 3, splitting the data into test and validation set are the initial parts.Separating data intotraining and testing sets is an important part of evaluating data mining models. Typically, when you separate a data set into a training set and testing set, most of the data is used for training, and a smaller portion of the data is used for testing [7]. Then we make the images as in form of vector form in order for the Neural Networks to perform the effective feature extraction. Here the images can't be given as raw instead they are formatted to vector form because CNN take input as raw csv that is the vector form in order for execution. As a part of the vector form is taken because $\mathrm{CNN}$ is the most advanced part in which it can take a small portion and iterate into multi-layers in which it keeps on extraction into several hidden layers. Each hidden layerhas an output to produce so as to give input the next layers.

So as features are extracted [2], those which are unwanted gets eliminated. But actually,it's not happening in CNN because, those unwanted parts are stored later on as the addition of vectors for a negative value. Inorder to avoid that negative value CNN back propagates back to these unwanted features and also again apply the same effect again so as to gain the final output [8]. This is a great advantage if machine learning in use of neural networks wont waste any features as moving on.Finally, as features are extracted, thereby gaining a top accuracy and also along with classified disease as output. Its main merits are that can identification of plant diseases undetectable by naked eye, giving an approximate of $96 \%$ and also giving us a précised diagnosis.

\section{TEST CASES}

Table 2: Test cases

\begin{tabular}{|c|c|c|c|c|}
\hline TCID & Leaves tested & Expected result & Actual result & Status \\
\hline 1 & $\begin{array}{l}\text { Fruit Leaves } \\
\text { (diseased) }\end{array}$ & $\begin{array}{l}\text { Desired disease } \\
\text { name }\end{array}$ & $\begin{array}{l}\text { Display disease } \\
\text { name }\end{array}$ & Successful \\
\hline 2 & \begin{tabular}{|l} 
Fruit leaves \\
(healthy)
\end{tabular} & Healthy & Display healthy & Successful \\
\hline 3 & $\begin{array}{l}\text { Vegetable leave } \\
\text { (diseased) }\end{array}$ & $\begin{array}{l}\text { Desired disease } \\
\text { name }\end{array}$ & $\begin{array}{l}\text { Display disease } \\
\text { name }\end{array}$ & Successful \\
\hline 4 & $\begin{array}{l}\text { Vegetable } \\
\text { leave(healthy) }\end{array}$ & Healthy & Display healthy & Successful \\
\hline 5 & $\begin{array}{l}\text { Grain } \\
\text { leave(diseased) }\end{array}$ & $\begin{array}{l}\text { Desired disease } \\
\text { name }\end{array}$ & $\begin{array}{l}\text { Display disease } \\
\text { name }\end{array}$ & Successful \\
\hline
\end{tabular}

In Table 2, it can be understandable that the model accepting what kind of inputs, such as fruits (Figure6), grains (Figure4), vegetable (Figure5). Here it will analyse the input and produce the exact disease that crop has and also its related graph. For the sake, the model also accepts the good crop images for testing, so that prediction is right or not.

\section{EXPERIMENTAL RESULTS}

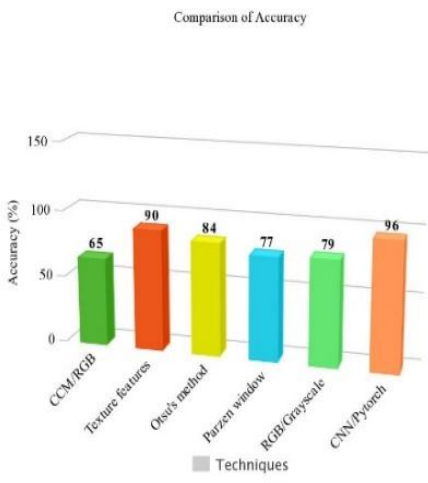

Figure 4: Result Analysis

Figure 4 gives an overview comparison of LIAM [3]. with respect to other methods that we take and the result is that LIAM [4]. is much more efficient in the case of accuracy and also int the implementation process. 


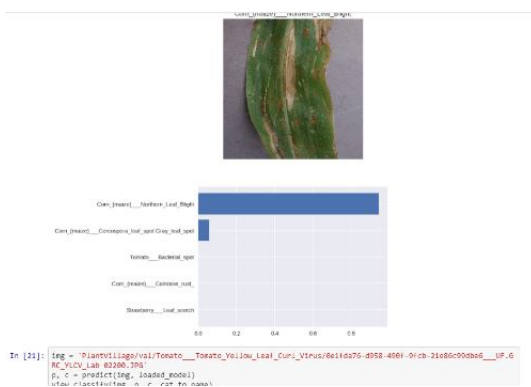

Figure 5: Output of LIAM (corn maize)

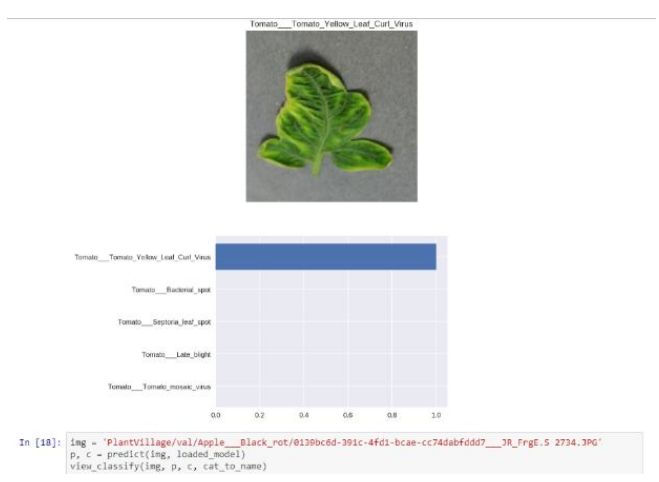

Figure 6: Output of LIAM (tomato)
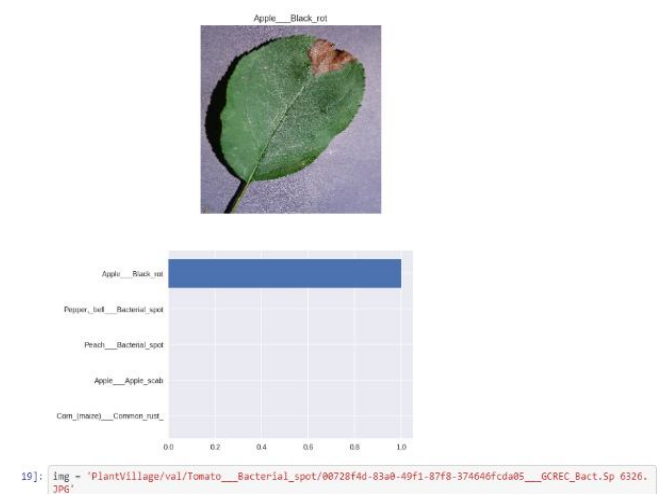

Figure 7 :Output of LIAM (apple)

\section{CONCLUSION AND FUTURE SCOPE}

The Results presented in this section are related to training with the whole database containing both original and augmented images. As it is known that convolutional networks are able to learn features when trained on larger datasets, results achieved when trained with only original images will not be explored. After fine-tuning the parameters of the network, an overall accuracy of $96 \%$ was achieved. Furthermore, the trained model was tested on each class individually. Test was performed on every image from the validation set. As suggested by good practice principles, achieved results should be compared with some other results.

In addition, there are still no commercial solutions on the market, except those dealing with plant species recognition based on the leaf's images. In this model, an approach of using deep learning method was explored in order to automatically classify and detect plant diseases from leaf images [6]. The complete procedure was described, respectively, from collecting the images used for training and validation to image pre-processing and augmentation and finally the procedure of training the deep CNN and fine-tuning. Different tests were performed in order to check the performance of newly created model. Future scope is that to make the system portable that is to make it accessible via different mediums like android, iOS, website etc. reduction in training time. It will be even more flexible inorder to work on different platforms and also finally produce even more accuracy.

\section{ACKNOWLEGEMENT}

We take this opportunity to express our sincere gratitude to all respected personalities who had guided and helped us in the completion of this paper. First, we express our thanks to Almighty God for guiding us in this venture and make it a huge success. We also thank the management of Mangalam College of Engineering, and Principal for permitting us to carry out this paper. Our sincere thanks to Head of Department of Computer Science and Engineering for permitting us to make use of facilities available in the department. Finally, we extend our gratefulness to all teaching and non-teaching staffs who were involved in this successful completion of this paper work and to all our friends who have patiently extended all sorts of help for accomplishing this paper.

\section{REFERENCES}

[1] Garima Tripathi, Jagruthi Save, An Image Pocessing And Neural Network Based ApproachFor Detection And Classification Of Plant Leaf Disease, IJCET, Volume 6, pp. 14-20, Issue 4, April (2015).

[2] Arivazhagan Selvaraj, Newlin Shebiah, Detection of unhealthy region of plant leaves and classification of plant leaf diseases using texture features, CIGR, Vol. 15, March (2013). 
[3] H. Al-Hiary, S. Bani-Ahmad, M. Reyalat, M. Braik and Z. ALRahamneh, Fast and Accurate Detection and Classification of Plant Diseases, IJCA, Vol. 17, March 2011.

[4] Wan Shung, Wan Shitong, Image Thresholding using weighted Parzen window estimation, Journal of Applied Science, Vol. 8, August 2011.

[5] K. Padmavati, K. Thangadurai, Implementation of RGB and Grayscale Images in Plant Leaves Disease Detection, IJST, Vol. 9, February 2016

[6] Shima Ramesh, Ramachandra Hebbar, Niveditha M.,Prasad Bhat N., Shashank N, Vinod PV., Plant Disease Detection Using Machine Learning,ICD13C, August 2018

[7] Meghana Govardhan; Veena M B, Diagnosis od plant disease using random forest, GCAT, October 2019

[8] Anubha Pearline Sundara Sobitha Raj, Sathiesh Kumar Vajravelu, Dual Deep learning architecture for plant species, IET, Vol13, July 2019

[9] Alaa Adel Araby, Mai Mohamed Abd Elhameed,Nada Mohamed Magdy ,Loa'a Ahmed Said ,Nada Abdelaal, Yomna Tarek Abd Allah,M. Saeed Darweesh, Mohamed Ali Fahim, Hassan Mostafa, Smart IOT monitoring system for agriculture and predictive analysis, MOCAST, July 2019 